\title{
Réanimations et Urgences
}

\author{
Jean-Jacques Lehot, Charles-Christian Arvieux. Collection « Le point sur ... » dirigée \\ par J.-L. Vincent et C. Martin. Springer-Verlag France. 2010, 546 pages. \\ ISBN 978-2-287-99128-8
}

\author{
André Denault, MD, PhD · Denise Fréchette, MD
}

Received: 23 February 2011/Accepted: 14 March 2011/Published online: 24 March 2011

(C) Canadian Anesthesiologists' Society 2011

Réanimation et urgences est un livre qui s'adresse à l'étudiant de médecine, le stagiaire ou l'infirmier(e) en stage d'urgence ou de réanimation. Cet ouvrage de 546 pages, sous la direction des docteurs Jean-Jacques Lehot et Charles-Christian Arvieux, a été rédigé par 58 auteurs de plusieurs régions de France. Le livre est divisé en quatre grandes sections, soit les pathologies, les terrains particuliers (pédiatrie, femme enceinte, personne âgée), les techniques et les questions professionnelles. La structure de soins d'urgence et de réanimation en France y est discutée au tout début. Chaque chapitre est codifié en fonction des sujets de l'examen national de médecine.

Les notions de base en réanimation sont expliquées de façon claire et concise. L'essentiel s'y retrouve. Il est parfois difficile toutefois de comprendre l'organisation intrinsèque de l'ouvrage étant donné qu'il n'existe pas au début des chapitres un plan de leur contenu avec les numéros des pages associées à chaque sous-titre. Par exemple, l'étudiant qui désirerait lire sur l'oxygénothérapie aurait peine à trouver que cette information se trouve en page 104. Il devrait se rendre au chapitre des pathologies respiratoires (pages 54 à 110) et feuilleter le chapitre au complet. L'index à la fin est aussi peu utile pour retrouver cette information. Le contenu du livre pourrait aussi bénéficier des nouvelles technologies de l'information. Par exemple, un étudiant en médecine pourrait mieux comprendre à l'aide d'un DVD ou d'une courte vidéo disponible sur un site web certaines techniques diagnostiques ou thérapeutiques comme l'insertion d'une voie centrale ou d'un drain thoracique. Ces éléments pourraient compléter l'information didactique comme il se fait dans l'enseignement de l'échographie.

En résumé, ce livre est bien écrit, pertinent dans l'enseignement en urgence et réanimation. Il présente un intérêt pour l'étudiant ou l'infirmier(e) francophone même si certains éléments du livre sont spécifiques à la formation et l'enseignement de la médecine en France.

\footnotetext{
A. Denault, MD, PhD ( $\triangle)$

Institut de cardiologie de Montréal, Montreal, QC, Canada

e-mail: andre.denault@umontreal.ca
}

A. Denault, $\mathrm{MD}, \mathrm{PhD} \cdot \mathrm{D}$. Fréchette, $\mathrm{MD}$

Université de Montréal, Montreal, QC, Canada 